(c) Elsevier/INRA

\title{
Le goupi (Goupia glabra Aubl), essence forestière d'avenir en Guyane : analyse bibliographique
}

\author{
JF Lacoste 1, DY Alexandre 2 \\ 1 Université de Paris XI, laboratoire d'écologie végétale, CNRS URA 121, \\ Bât 362, 91405 Orsay Cedex; \\ 2 ORSTOM, 213 rue Lafayette, 75480 Paris Cedex 10, France
}

(Reçu le 21 septembre 1990; accepté le 20 mars 1991)

\begin{abstract}
Résumé - Le goupi (Goupia glabra Aubl) est une essence forestière tropicale à croissance rapide et aux caractéristiques intéressantes. II présente une remarquable plasticité aux conditions écologiques et notamment aux contraintes édaphiques. Grâce à la physiologie de ses graines qui lui donnent des caractéristiques d'espèce pionnière, le goupi peut envahir rapidement les surfaces mises à nu. L'arbre présente un fût droit et son bois naturellement résistant convient à de multiples usages. Les expériences conduites en Guyane française ont montré que le dégagement précoce de jeunes recrûs forestiers est une méthode sylvicole prometteuse pour obtenir des peuplements enrichis intéressants pourvu que le stock initial de semis naturels soit suffisant.
\end{abstract}

\section{Goupia glabra / sylviculture / enrichissement / écologie / forêt tropicale}

Summary - Kopi (Goupia glabra Aubl), a promising timber tree for forestry: a literature review. Kopi (Goupia glabra Aubl) is a fast growing tropical timber tree with interesting features. It exhibits a tremendous versatility to ecological conditions, and notably to soil constraints. Owing to its seed ecophysiology and pioneer characteristics, kopi is able to invade open areas rapidly. The tree possesses a straight bole and its wood, which is naturally long-lasting, is suitable for most purposes. The authors suggest an ecological approach to sylviculture, based mainly on experiments conducted on secondary regrowth in French Guiana. These experiments have shown that the clearing of the early stage of forest regrowth (ie, the removal of a group of undesirable secondary species, eg Cecropia spp, Vismia spp and Solanum spp) could lead to an enriched forest. It has also been noticed that kopi planting could be beneficial for reforestation if economic constraints were not so severe. In this case a nursery of kopi would be the main problem. Finally, it is concluded that early clearing is a promising way of obtaining a valuable stand of secondary forest provided that the initial regrowth is sufficiently rich in timber tree seedlings.

Goupia glabra / sy/viculture / enrichment / ecology / tropical forest 


\section{INTRODUCTION}

Face à la déforestation rapide des forêts tropicales et notamment du massif amazonien, la sylviculture devient une nécessité de plus en plus pressante. Pendant longtemps, l'effort des forestiers, particulièrement en Afrique francophone, s'est concentré sur la technique des plantations en plein. Malgré des réussites techniques incontestables, ces plantations très coûteuses ne répondent pas aux besoins économiques des pays concernés. On se tourne actuellement davantage vers l'amélioration des peuplements naturels dont la rentabilité, compte tenu des rôles multiples de la forêt, apparaît plus sûre sinon meilleure (Leslie, 1987).

Ainsi, les forêts secondaires plus ou moins dégradées qui remplacent progressivement les forêts primaires, renferment des espèces technologiquement intéressantes, qu'une sylviculture adaptée pourrait favoriser.

Dans les forêts secondarisées en Guyane, on rencontre ainsi, pour ne citer que les espèces les plus fréquentes: la bagasse (Bagassa tiliaefolia), le bourgoni (Inga bourgoni), le carapa (Carapa spp), le copaî́a (Jacaranda copaia), le simarouba (Simarouba amara) et le goupi (Goupia glabra). Cette dernière espèce est considérée depuis longtemps comme particulièrement intéressante. Des rares travaux qui lui ont été consacrés, on retiendra parmi les plus classiques ceux de Schulz (1960) au Surinam qui montrent l'excellente réponse de l'espèce aux traitements de dégagement. Les travaux que nous avons conduits sur le bassin «D» dans le cadre du programme MAB ECEREX apportent des données récentes sur le comportement de cette espèce en conditions naturelles (Lacoste et Alexandre, 1989; Lacoste, 1990; Lacoste et Alexandre, 1990).
Nous présentons ici une synthèse des données bibliographiques et de notre expérience sur cette essence.

\section{CARACTĖRES GÉNÉRAUX DE GOUPI, LE BOIS, SON COMMERCE}

\section{Systématique - critères de reconnaissance - inorphologie - modèle architectural}

Goupia glabra Aubl est connu sous le nom de goupi en Guyane française, kopie au Surinam, cupiuba ou tento au Brésil, congrio blanco au Vénézuéla. C'est un très grand arbre appartenant à la famille des Celastraceae ou parfois classé dans une famille propre : les Goupiaceae Miers (cf Willis, 1973), ce qui indique bien son caractère particulier. Aublet qui décrivit le premier l'espèce en 1775 lui attribua deux binômes latins, aujourd'hui synonymes : Goupia glabra décrit sur la forme adulte et Goupia tomentosa sur la forme juvénile. En effet, les deux stades diffèrent profondément. La forme adulte a de petites feuilles vernissées, épaisses et cassantes, tandis que les jeunes individus présentent des feuilles plutôt grandes, poilues, dentées et à vrai dire très variables pour tous les caractères comme la couleur, l'épaisseur ou la forme. Dans tous les cas ce sont des feuilles simples, entières (lancéolées) et alternes. Un autre synonyme de l'espèce est Goupia paraensis Hub (Lemée, 1953).

L'arbre est sempervirent mais d'après Alencar et al (1979), il y a un pic dans la chute de feuilles en saison sèche (cfinfra).

Les fleurs très petites sont groupées en ombelles. Elles sont hermaphrodites mais on n'a aucune indication sur leur biologie reproductrice. Elles sont d'une couleur 
jaune-verdâtre, très discrètes et donc probablement anémophiles.

Le fruit est une petite baie sphérique d'environ $5 \mathrm{~mm}$ de diamètre, de couleur rouge-orangé à maturité. II contient de 5 à 10 graines (Prévost, 1983), voire davantage, mais rarement plus d'une graine viable. Ce sont des graines arillées, albuminées, très petites, mesurant environ $2 \mathrm{~mm}$ de long et $1 \mathrm{~mm}$ de large et pesant environ $1,6 \mathrm{mg}$.

La plantule est épigée au sens de Miquel (1987), de très petite taille $(<3 \mathrm{~mm}$ ) et possède des cotylédons foliacés comme beaucoup d'espèces héliophiles.

En forêt, l'arbre se reconnaît bien grâce à son port caractéristique lié à un feuillage très clair et à une ramification très anguleuse. La hauteur de l'arbre peut dépasser 50 mètres (Oldeman, 1974) et le fût cylindrique peut atteindre $25 \mathrm{~m}$ de haut et 1,20 à $1,30 \mathrm{~m}$ de diamètre. II est muni à la base de contreforts épais mais peu larges pouvant s'élever jusqu'à $2 \mathrm{~m}$. Des racines traçantes sont parfois apparentes jusqu'à $5 \mathrm{~m}$ de distance (Bena, 1960). L'écorce rugueuse est fendillée dans le sens vertical et localement écaillée. Elle est de teinte gris-argenté ou gris-rosâtre (Thiel, 1983). D'après Rooth (1972), cette écorce présente la particularité de posséder de nombreuses cellules pierreuses. Les jeunes individus ont un port caractéristique "en sapin de Noël» qui correspond au modèle architectural de Roux (Hallé et Oldeman, 1970). L'espèce rejette de souche très abondamment et peut former des cépées. Les racines sont endomycorrhiziennes (Bonetti et al, 1983).

\section{Caractères technologiques, usages et valeur commerciale du bois}

Comparativement à bien d'autres essences tropicales le goupi est dans l'ensemble bien conformé : bille droite, longue, sans nœuds ni cœur creux. L'aubier est peu différencié. Le rendement au sciage est de 54\% (Sudam, 1979), ce qui est bon.

Le bois est de couleur brun clair avec des reflets roses. II possède un fil bien droit, se travaille facilement et prend un très beau poil qui le rend propre aux usages de l'ameublement. Le bois fraîchement coupé a une odeur forte, désagréable pour certains, qui disparaît ou s'atténue au séchage. La densité sèche à l'air (12\% d'humidité) est de 0,85 (Bena, 1960). Le bois possède une bonne cohésion transversale et un faible retrait. Sa structure a été étudiée par Araujo et Filho (1973) ainsi que par Detienne et al (1982). II résiste naturellement à la pourriture et aux termites, ce qui le destine aux usages de menuiserie et de charpentes extérieures $y$ compris en milieu marin (pontons).

Les populations tribales de Guyane utilisent le goupi pour la confection des coques des pirogues. Notons également que l'écorce a des propriétés analgésiques couramment utilisées par ces populations pour combattre les maux de dent (Grenand et al, 1987).

En raison de son abondance et pour toutes ses propriétés, le goupi tient une place importante sur le marché régional du bois. C'est la sixième essence de Guyane (Détienne et al, 1990) et la première du Surinam (Vink, 1977). Au Brésil, il est une des principales essences commerciales de l'état du Para (Dubois 1978, in Rollet 1983).

\section{CARACTĖRES ÉCOLOGIQUES DU GOUPI : DYNAMIQUE EN FORÊT GUYANAISE}

\section{Répartition géographique}

L'aire géographique du goupi recouvre l'ensemble de la région des Guyanes, de 
la Colombie et du Vénézuéla jusqu'au Brésil (Béna, 1960) et s'étendrait même en Amazonie jusqu'aux contreforts des Andes selon Hallé (comm pers). Lescure (1986) indique que dans la région de Trois-Sauts au sud de la Guyane, le goupi est l'arbre qui domine la forêt. Dans la région de Curua Una au Brésil, selon Rollet (1983), c'est la seconde espèce en fréquence pour les arbres de plus de $60 \mathrm{~cm}$ de diamètre. Au Surinam, le goupi forme des peuplements quasi monospécifiques (Schulz, 1960).

En Guyane française, le goupi est actuellement totalement absent de "l'ile" de Cayenne où selon Aublet ou Lemée (loc cit) il était jadis présent. C'est probablement là le résultat d'une secondarisation excessive du milieu.

\section{Structure de population}

D'après Schulz (1960), la distribution diamètrale du goupi en forêt primaire au Surinam montre souvent un déficit marqué dans les classes de petits et/ou moyens diamètres. En Guyane, Gazel (1982) souligne également le déficit en forêt primaire des tiges de $30 \mathrm{~cm}$ de diamètres (fig 1). Ce type de distribution, qui ne traduit pas un problème d'échantillonnage, est caractéristique au contraire des espèces fortement héliophiles (voir aussi Rollet, 1969 et 1983). Le goupi est en effet une espèce qui ne régénère que dans les trouées.

La distribution erratique ou très étalée des fréquences au-dessus de $30 \mathrm{~cm}$ de diamètre indique que la mortalité des individus qui se maintiennent en pleine lumière est faible jusqu'à un âge avancé. La mortalité dans les jeunes stades est en revanche élevée, elle atteint par exemple $21,3 \%$ par an entre 5,5 et 7 ans dans un recrû du dispositif ECEREX, le bassin "D"
(Lacoste, 1990). Cela traduit les conséquences de la forte compétition avec les espèces pionnières à vie courte et croissance très rapide (eg Cecropia spp).

\section{Phénologie}

La phénologie n'est pas bien connue. Les périodes de floraison, de fructification ou de chute des feuilles varient dans la littérature selon les auteurs et les sites d'observation (Bena, 1960; Alencar et al, 1979; Ayphassorho, 1984; Sabatier, 1983; etc). En fait, au petit nombre d'individus suivis (généralement inférieur à 10) et à la durée
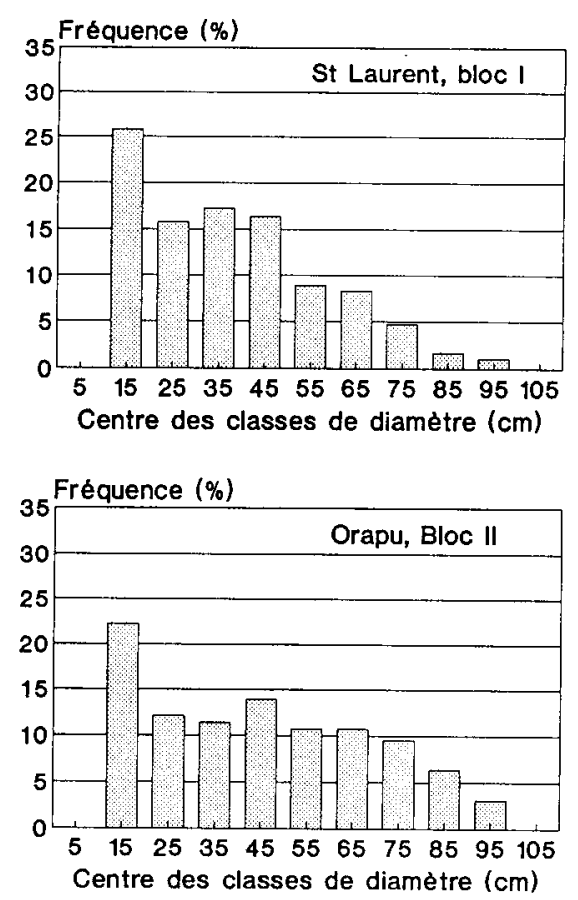

Fig 1. Structure de populations naturelles de goupi dans deux sites de forêt primaire en Guyane française, pour les arbres de plus de $30 \mathrm{~cm}$ de diamètre (d'après Gazel, 1983). 
différente des études, s'ajoute la grande variabilité annuelle des conditions climatiques. Ces facteurs ne rendent compte qu'en partie au moins des variations des données disponibles. Ainsi, bien que le goupi puisse présenter un maximum de floraison et de défoliaison en saison sèche, la grande variabilité des données publiées apparaît bien réelle. En effet, d'après nos observations, il n'y a pas de synchronisme entre les divers individus d'une population et on peut même observer différents stades phénologiques au sein d'un seul houppier.

Au total, la floraison et par conséquent la fructification sont étalées sur l'ensemble de l'année (fig 2).

\section{Dispersion}

Les fruits sont consommés par de nombreuses espèces d'oiseaux dont certains, surtout les petits, sont connus pour être de bons disséminateurs de graines ( $c f$ tableau I ex Lacoste, 1990, p 33).

\section{Graines dans le sol}

Les graines semblent posséder une dormance de type photolabile. Ce type de dormance qui empêche la germination tant que le couvert reste continu permet aux graines de s'accumuler dans le sol et de ne germer que dans les ouvertures. C'est

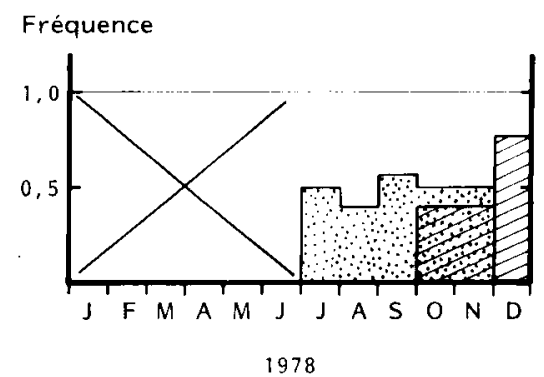

Fréquence

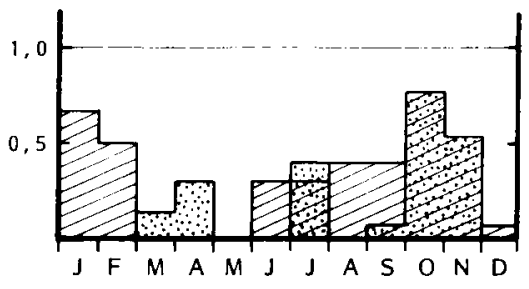

1979

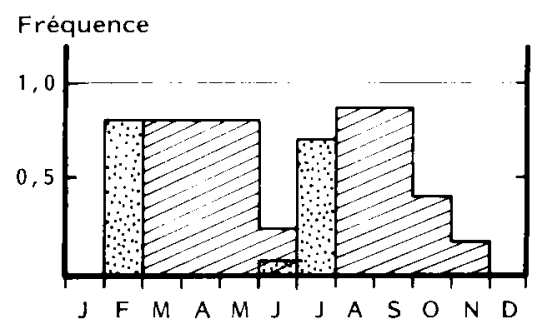

1980

Fréquence

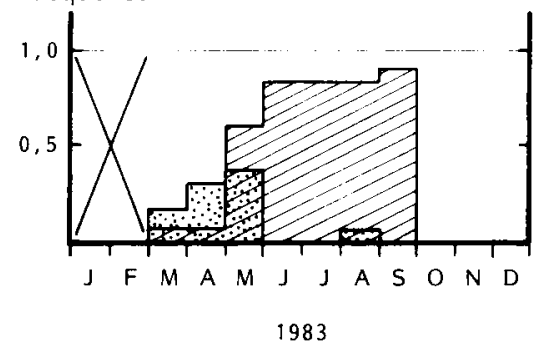

Fréquence de floraison
Fréquence de fructification

Fig 2. Évolution de la fréquence mensuelle de floraison et de fructification du goupi en forêt primaire (calculée sur 13-14 arbres, d'après Ayphassorho, 1984). 
Tableau I. Quelques oiseaux consommateurs de fruits de Goupia glabra, Piste de St Elie (Lacoste, 1990).

$\begin{array}{ll}\text { Columba plumbea } & \text { Tangara chilensis } \\ \text { Cotinga cotinga } & \text { Tangara mexicana } \\ \text { Florisuga mellivora } & \text { Throupis episcopus } \\ \text { Lipeugus vociferans } & \text { Trogon viridis } \\ \text { Pipra erythrocephala } & \text { Turdus albicollis } \\ \text { Pipra pipra } & \text { Xipholena punicea }\end{array}$

Ramphocelus carbo jeunes feuilles atteint en moyenne $14 \mathrm{~cm}$ de long et une surface de plus de $40 \mathrm{~cm}^{2}$ (Rollet (1969) cite pour des feuilles adultes une valeur moyenne de $24,3 \mathrm{~cm}^{2}$ ), tandis que son épaisseur moyenne est de $160 \mu \mathrm{m}$. La masse surfacique augmente avec l'âge et l'ensoleillement. Nous avons mesuré sur des individus de 7 ans des valeurs extrêmes de $38 \mathrm{~g} / \mathrm{m}^{2}$ pour des feuilles de sous-bois à plus de $120 \mathrm{~g} / \mathrm{m}^{2}$ pour des feuilles de la voûte, ce qui est proche des caractères de feuille adulte. Sur le plan expérimental, Colin (1989) montre que la régulation stomatique est très sensible au déficit de saturation de l'air même lorsque l'alimentation hydrique est satisfaisante. Selon Alexandre (1991), l'espèce se montre tolérante au déficit hydrique : elle transpire en saison sèche malgré un bilan déficitaire. La régulation stomatique est progressive à mesure que le déficit hydrique s'accentue, et bien que la teneur en eau des feuilles puisse s'abaisser fortement, on n'observe aucune lésion physiologique (tableau II).

Le caractère dominant de l'espèce est sans doute son étonnante plasticité à la lumière comme l'ont bien montré Alencar et Araujo (1980). L'espèce est en effet héliophile, mais si elle pousse mieux en plein soleil, elle peut supporter des ombrages très denses. Lacoste (1990) montre sur le bassin « $\mathrm{D}$ » à Ecerex l'excellente réponse de l'espèce (croissance fortement stimulée, mortalité diminuée d'un facteur 3,1 ) à une mise en lumière modérée précoce ( $c f$ infra).

La croissance en hauteur est très variable. À Ecerex, en milieu ouvert, nous avons mesuré des croissances maximales supérieures à trois mètres par an pendant les premières années.

La croissance en diamètre est également très variable. Sur le bassin "l» d'Ecerex, très éclairci, nous avons mesuré une croissance moyenne en diamètre sur 2,5

L'écophysiologie de l'espèce est encore très mal connue. Les feuilles sont hypostomatiques et présentent des densités stomatiques de $500 / \mathrm{m}^{2}$. Les stomates ont une longueur de $18 \mu \mathrm{m}$. Le limbe des 
Tableau II. Comportement hydrique en saison sèche du goupi (Goupia glabra comparé à un arbre strictement héliophile, le tréma (Trema guianensis) et un arbre sciaphile, le wapa (Eperua falcata) (d'après Alexandre, 1991).

\begin{tabular}{|c|c|c|c|}
\hline & Tréma & Goupi & Wapa \\
\hline $\begin{array}{l}\text { Potentiel hydrique de base } \\
\text { Potentiel hydrique mini } \\
\text { Conductance stomatique maxi } \\
\text { Conductance cuticulaire } \\
\text { Régulation osmotique } \\
\text { Teneur en MS des feuilles } \\
\text { Déficit de saturation à la } \\
\text { perte de turgescence } \\
\text { Déficit de saturation sub-létal } \\
\text { Fanaison (rigidité des feuilles) }\end{array}$ & $\begin{array}{l}\text { élevé } \\
\text { élevé } \\
\text { élevée } \\
\text { élevée } \\
\text { faible } \\
\text { faible } \\
\text { élevé } \\
\text { fort moyen } \\
\text { rapide }\end{array}$ & $\begin{array}{l}\text { bas } \\
\text { très bas } \\
\text { faible } \\
\text { élevée } \\
\text { faible } \\
\text { moyenne } \\
\text { moyen } \\
\text { faible } \\
\text { lente }\end{array}$ & $\begin{array}{l}\text { moyen à bas } \\
\text { moyen à bas } \\
\text { faible } \\
\text { faible } \\
\text { moyenne } \\
\text { élevée } \\
\text { faible } \\
\text { non observée }\end{array}$ \\
\hline
\end{tabular}

ans de 0,8 0,1 cm par an. Sur le même bassin, le maximum observé est de 2,72 $\mathrm{cm}$ par an à 8 ans. Sur la bassin "D», plus dense que le bassin «l», Lacoste (1990) observe dans un recrû de 1,5 ans une croissance moyenne en circonférence de $2,8 \mathrm{~cm} /$ an la première année après un traitement de dégagement sélectif léger, alors qu'elle n'est que de $1,0 \mathrm{~cm} / \mathrm{an}$ en zone témoin (fig 3). La troisième année après dégagement, la croissance moyen-ne en zone dégagée n'est plus que de 1,4 cm/an. Après trois ans, la refermeture du couvert fait que la majorité des tiges ne poussent plus; les maximums observés restent cependant importants avec $6,0 \mathrm{~cm}$ après quatre ans et encore $2,0 \mathrm{~cm}$ après 5,5 ans (fig 4).

Dans une plantation de 11 ans au Brésil, Pedroso et Pereira (1971) observent une hauteur totale moyenne de $15,4 \mathrm{~m}$ pour un diamètre moyen de $15,5 \mathrm{~cm}$. La production en volume sur cette période atteint $30 \mathrm{~m}^{3} / \mathrm{ha}$ par an. La production moyenne à 15 ans de cette plantation reste supérieure à $16 \mathrm{~m}^{3} / \mathrm{ha}$ par an (Sudam, 1979), ce qui reste tout à fait considérable.
Des expériences en pots sous serres (Bariteau et al, 1990) montrent que la croissance en diamètre et en hauteur du goupi est meilleure sous $25 \%$ d'éclairement relatif que sous des éclairements supérieurs ou inférieurs. Ces résultats sont difficilement conciliables avec les meilleures croissances observées in situ dans les situations les plus dégagées. Si le tempérament plastique généralement reconnu au goupi peut rendre compte de ces variations, le conditionnement en pot et sous ombrière entraîne aussi peut-être des modifications du comportement de l'espèce (problème de mycorrhization, confinement des racines...). La généralisation des résultats de ces expériences aux conditions naturelles doit donc être très prudente.

\section{Croissance des adultes}

À l'âge adulte, la croissance du goupi reste extrêmement variable comme l'a bien montré Schulz (1960). Le maximum observé par cet auteur atteint $2,5 \mathrm{~cm}$ de circonfé- 


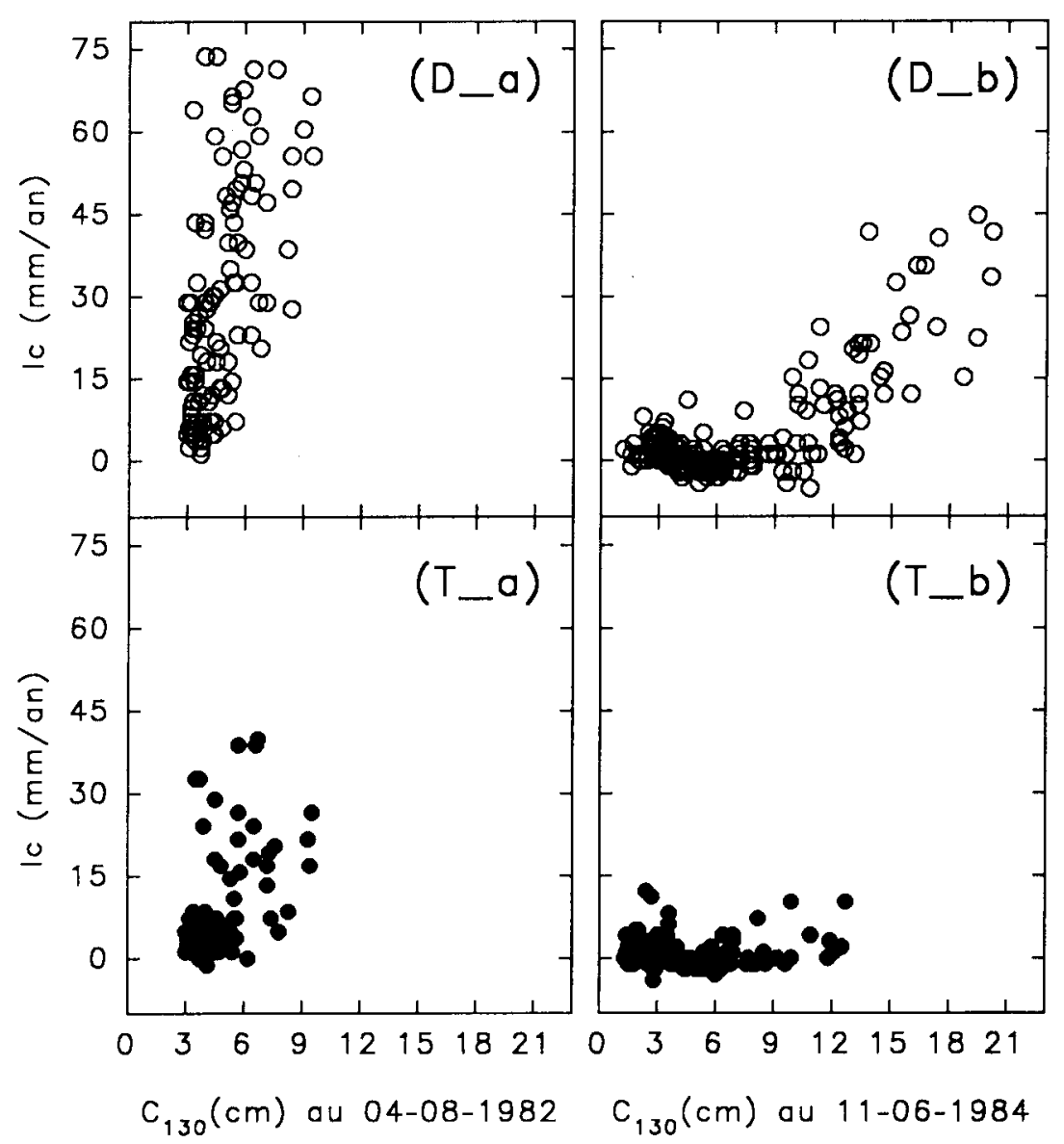

Fig 3. Croissance annueile en circonférence du goupi dans un jeune recrû forestier : (D_a) 1 an après un dégagement sélectif précoce, ( $\mathrm{D}$ b $\mathrm{b}) 3$ ans après un dégagement sélectif précoce, comparée avec le témoin, respectivement (T_a) et (T_b) (Lacoste, 1990).

rence par an, la moyenne se situant entre 1,0 et $1,5 \mathrm{~cm}$ par an.

En Guyane française, Gazel (1983) mesure dans les parcelles du Bafog une croissance en diamètre moyenne "Soutenue" de 5 à $8 \mathrm{~mm} / \mathrm{an}$ selon les parcelles, avec des maximums atteignant 12 à $15 \mathrm{~mm}$. À titre de comparaison, les valeurs citées par cet auteur dans les mêmes conditions pour l'angélique (Dicorynia guianensis Caesalpiniacées) et le gonfolo (Qualea rosea Vochysiacées), espèces réputées productives, sont respectivement de 6 et $7 \mathrm{~mm} / \mathrm{an}$, avec des maximums identiques. Les performances du goupi apparaissent ainsi comme bonnes et justifient tout l'intérêt que l'on doit porter à cette espèce. 

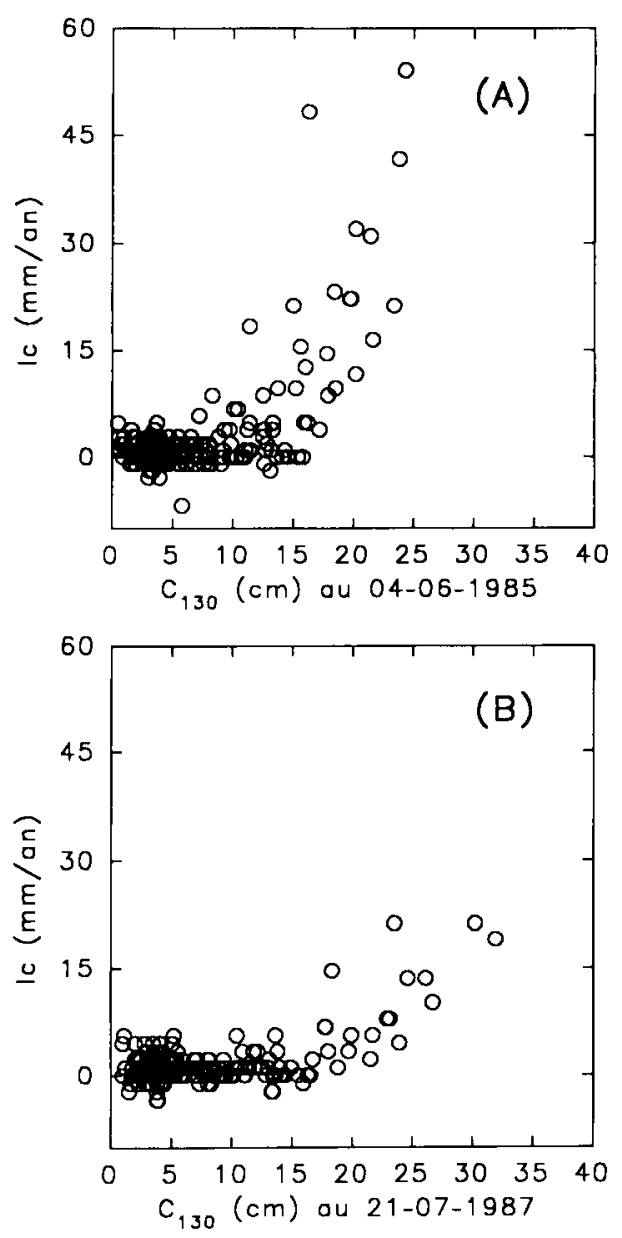

Fig 4. Croissance annuelle en circonférence du goupi dans un jeune recrû forestier: (A) 4 ans après un dégagement sélectif précoce, (B) 5,5 ans après un dégagement sélectif précoce (Lacoste, 1990).

\section{Exigences édaphiques}

L'espèce est également très plastique à l'égard du sol. Aubreville (1961) indique qu'on la rencontre sur des sols plutôt frais et lourds, tandis que Béna (1960) la si- gnale surtout sur des terrains sableux et bien drainés, mais ces auteurs ne donnent pas de précision sur les données utilisées. Cependant, Loureiro et Silva (1968) signalent bien qu'on la trouve indifféremment sur ces deux types de substrats. Nos propres observations sur les premiers stades de la régénération ont montré que les capacités de croissance de l'espèce n'étaient pas affectées par des conditions de drainage très contrastées (Lacoste, 1990; Lacoste et Alexandre, 1990). Des observations préliminaires sur le système radiculaire ont en effet montré que l'espèce franchit aisément la barrière physique de drainage rencontrée dans les sols guyanais (Alexandre, 1988). Alors que ces derniers sont ainsi physiquement très contraignants (Boulet, 1978), cette caractéristique fait du goupi une espèce bien adaptée aux contraintes locales.

\section{Sylviculture et avenir du goupi}

En zone tropicale humide, la complexité de l'écosystème forestier rend difficile la mise au point de méthodes sylvicoles performantes. De nombreuses études expérimentales sont en cours (notamment en Guyane) qui visent à une meilleure gestion de la forêt naturelie au bénéfice des essences précieuses. Le tempérament de ces dernières ne permet bien souvent que l'application de méthodes extensives. II apparaît cependant qu'une sylviculture intensive peut être développée au profit des essences héliophiles de valeur. Le goupi en est une. Toutefois, les études qui lui ont été consacrées, n'ont pas encore conduit à une véritable méthode sylvicole la concernant. Les paragraphes précédents permettent de dégager des éléments qui pourraient être à la base d'une sylviculture du goupi et plus largement des espèces secondaires tardives (au sens de Budowski, 1965). S'il existe des arguments en faveur 
de la régénération artificielle de cette espèce, c'est en revanche la régénération naturelle qui correspondrait le mieux aux objectifs de valorisation des formations secondaires et aux potentialités de l'espèce. C'est donc ce premier point que nous aborderons tout d'abord.

De par ses caractéristiques d'espèce pionnière, la régénération du goupi n'intervient que dans les trouées (chablis ou exploitation forestière) ou les coupes rases. C'est alors le degré de perturbation de la forêt initiale qui détermine l'abondance des semis. Celle-ci est grande (mais dépend du nombre de semencier initiaux) dans les recrûs après coupe de la forêt primaire, mais elle décroît rapidement quand la secondarisation des peuplements de départ augmente. On peut ainsi envisager, comme le suggère Rollet (1983), de conserver des semenciers d'essence héliophiles dans les peuplements soumis à l'exploitation pour maintenir une pression de colonisation de ces espèces.

La très forte mortalité due à la compétition dans les très jeunes stades et les potentialités de forte croissance juvénile dans de bonnes conditions imposent une intervention sylvicole rapide dans les recrûs de manière à préserver l'avenir de la régénération. Nos essais conduits à Ecerex montrent que la valeur sylvicole des recrûs forestiers naturels peut ainsi facilement être améliorée par un dégagement précoce des individus des espèces intéressantes. L'intervention sylvicole consiste ici à supprimer, en une seule fois par abattage, dans un recrû (issu de coupe papetière) de 1,5 an les espèces pionnières les plus compétitives et sans valeur commerciale actuelle (ie, Cecropia spp, Vismia spp et Solanum spp). Les résultats indiquent que cette intervention a un effet positif durable tant sur la croissance du goupi qui est stimulée que sur la mortalité qui est fortement diminuée. Ici, la faible intensité du dégagement a également eu pour effet de ne pas provoquer la prolifération des lianes, toujours à craindre dans ce type de milieu et de contribuer efficacement à l'éducation des tiges en favorisant l'élagage.

Ces résultats tangibles montrent tout l'intérêt du modèle sylvicole proposé. Les études déjà évoquées portant sur les placeaux de Bafog (Gazel, 1983) suggèrent qu'il trouverait de plus un excellent champ d'application dans les grandes trouées consécutives à l'exploitation forestière. Les expérimentations à poursuivre dans ce domaine devraient donc s'attacher à préciser le nombre et l'intensité optimale des traitements de dégagement à réaliser et donc les coûts. Dans cette optique, il faudrait aussi déterminer la densité de semis d'essences de valeurs en dessous de laquelle l'intervention sylvicole n'est pas applicable.

On peut aussi envisager une sylviculture basée sur les techniques de la régénération artificielle. À ce titre, la bonne adaptation du goupi aux contraintes pédologiques locales rend l'espèce particulièrement intéressante. La très forte productivité de cette espèce en plantation, révélée par des études expérimentales (Pedroso et Pereira, 1971; Sudam, 1979) est un atout supplémentaire. Cependant, ces études expérimentales n'ont pas été, à notre connaissance, suivies d'essais en vraie grandeur. À ce niveau, la grande difficulté est en fait l'obtention des graines pour les semis en pépinière. En effet, la consommation des fruits mûrs par les oiseaux, la petite taille des fruits et la grande taille des semenciers sont autant d'obstacles à la récolte de graines en quantité suffisante. On peut alors envisager la création de vergers semenciers avec des porte-graines greffés ou la technique du bouturage ou tout autre procédé de multiplication végétative. Nos essais préliminaires ont mis en évidence la difficulté du bouturage, mais aussi les bonnes potentialités du marcottage (non publié). 
Un problème à ne pas négliger en plantation est celui des attaques de ravageurs. Le goupi est très sensible à l'attaque des fourmis champignonistes (Foggie, 1960; Foresta, 1983) qui, si elle n'est pas létale pour l'espèce, peut compromettre ou tout au moins ralentir la croissance des jeunes individus.

En plantation, en plein découvert, on peut également craindre la mauvaise conformation des sujets due à la mort de l'apex. Celle-ci est en effet fréquente dans ces conditions et est suivie de la formation de tiges multiples. Nous avons observé sur le bassin «|». Ecerex, en conditions naturelles, qu'en fait, une sélection spontanée et rapide s'opère qui élimine les brins surnuméraires et restitue une bonne conformation à la tige.

Cependant, les critères de rentabilité économique sont aujourd'hui davantage en faveur de la régénération naturelle (Leslie, op cit). La Guyane, où les coûts de maind'œuvre sont élevés, n'échappe pas à ce constat. De sorte que même si les problèmes techniques qu'elle pose ne sont pas insurmontables, la régénération artificielle de cette espèce n'a que peu d'avenir actuellement dans le département. En revanche, le modèle sylvicole que nous proposons nécessite peu d'intrants. II s'intègre dans le cadre d'une meilleure gestion des régénérations naturelles et vise à l'obtention d'une augmentation de production des essences héliophiles de valeur. Or, on sait que la valeur d'une essence forestière repose autant sur les propriétés de son bois que sur la quantité et la régularité de sa production. Ces derniers augmentant, on peut penser que la place du goupi sur le marché international du bois augmentera dans le même sens.

\section{CONCLUSION}

Le goupi apparaît comme une espèce de grande valeur sylvicole. Elle réunit en effet de nombreuses particularités intéressantes, notamment:

- une plasticité écologique importante permettent sa large répartition;

- une excellente adaptation aux sols contraignants rencontrés en Amazonie;

- une aptitude à envahir précocement les zones ouvertes. Le caractère d'essence pionnière étant lié à l'écophysiologie des graines;

- une héliophilie marquée qui la prédispose à une sylviculture semi-intensive;

- une croissance très rapide dans de bonnes conditions;

- une conformation potentiellement favorable;

- enfin et surtout, un bois aux très grandes qualités technologiques malgré une odeur qui peut exiger des précautions de mise en œuvre.

Cette espèce présente l'inconvénient principal d'être insuffisamment connue en dehors du marché local.

La grande variabilité écologique rencontrée en forêt peut certes apparaître comme un obstacle au développement immédiat de sa sylviculture mais elle constitue en fait un atout pour l'avenir en rendant possible une sélection rapide d'individus performants.

Les résultats que nous avons obtenus montrent d'autre part que cette espèce peut être utilisée dans un modèle simple de sylviculture pour valoriser les formations secondaires et participer ainsi à une meilleure gestion des forêts tropicales. 


\section{RÉFÉRENCES}

Alencar JDC, Almeida RA, Fernades NP (1979) Fenologia de espécies florestais em floresta tropical umida de terra firme na Amazônia Central. Acta Amazonica 9 (1), 163-198

Alencar JC, Araujo VC (1990) Comportamento de espécies florestais amozonicas quanto à luminosidade. Acta Amazonica 10 (3), 435444

Alexandre DY (1988) Note : aperçu sur la morphologie radiculaire de deux espèces pionnières de Guyane française : Goupia glabra et Vismia guianensis. Multigraphie Orstom, $5 p$

Alexandre DY (1989) Dynamique de la régération naturelle en forêt dense de Côte d'lvoire: stratégies écologiques des arbres de la voûte et potentiels floristiques. Collection Études et Thèses, Orstom, Paris, $102 \mathrm{p}$

Alexandre DY (1991) Comportement hydrique au cours de la saison sèche et place dans la succession de trois arbres guyanais: Trema micrantha, Goupia glabra et Eperua falcata. Ann Sci For 48, 101-112

Araujo PAM, Filho AM (1973) Estrutura da madeira de Goupia glabra Aubl (Goupia-Ceae). Arq Jard Bot do Rio 19, 149-153

Aublet $F$ (1775) Histoire des plantes de la Guyane française. Réédition 1977, Cramer, Vaduz, 2 vol

Aubreville A (1961) Aperçu sur la flore de la Guyane française. Bois For Trop 80, 3-12

Ayphassorho H (1984) Étude de la croissance en circonférence et des rythmes phénologiques de quelques essences guyanaises. Multigraphie CTFT, Kourou, $99 \mathrm{p}$

Bariteau M, Huc R, Montpied P (1990) Évolution de l'impact de traitements sylvicoles en forêt tropicale humide sur la régénération; illustration à partir du comportement in situ et en conditions contrôlées de deux expèces de Guyane. Atelier MAB de Cayenne, mars 1990, $10 p$

Béna P (1960) Essences forestières de Guyane. Bureau Agricole et Forestier Guyanais, Paris, $469 p$

Bonetti R, Oliveira LA, Magalhaes FMM (1983) Populaçao de rhizobium ssp e ocorrencia de micorriza VA em cultivos de essencias florestais. Pesqui Agropecu Bras $10 \mathrm{~s} / \mathrm{n} 137-$ 142

Boulet R (1978) Existence de systèmes à forte différenciation latérale en milieu ferralitique guyanais : un nouvel exemple de couverture pédologique en déséquilibre. Sci Sol, Bull AFES (2), 75-82

Budowski G (1965) Distribution of tropical American rain forest species in the light of successional process. Turrialba 15 (1), 40-42

Colin F (1989) Écophysiologie en forêt équatoriale. Contribution à la connaissance de Goupia glabra. Rapport thème $1^{\text {re }}$ année ICF, ENGREF, Kourou, $37 \mathrm{p}$

Détienne $P$, Jacquet $P$, Mariaux A (1982) Manuel d'identification des bois tropicaux. Tome 3, Guyane française, CTFT, $321 \mathrm{p}$

Détienne P, Fouquet D, Parant B (1990) Les bois guyanais, propriétés et utilisation. Bois For Trop 219, 125-143

Dubois J (1978) Curso multinacional de capacitacao em sylvicultura e manejo de florestas amazônicas, Belterra. Curua Una Memeogr $128 \mathrm{p}$

Foggie A (1960) Natural regeneration in the humid tropical forest. Caribb For 21, 73-81

Foresta $\mathrm{H}$ de (1983) Hétérogénéité de la végétation pionnière en forêt tropicale humide : exemple d'une coupe papetière en forêt guyanaise. Oecol Applic 4 (3), 221-235

Gazel M (1983) Croissance des arbres et productivité des peuplements en forêt dense équatoriale guyanaise. Multigraphie ONF, Cayenne, $302 \mathrm{p}$

Grenand P, Moretti C, Jacquemin H (1987) Pharmacopées traditionnelles en Guyane; Créoles, Palikur, Wayapi. Collection Mémoires $n^{\circ} 108$, Orstom, Paris, $569 p$

Hallé F, Oldeman RAA (1970) Essai sur l'architecture et la dynamique de croissance des arbres tropicaux. Masson, Paris, $178 p$

Lacoste JF (1990) Effets de la suppression d'arbres pionniers sur l'évolution d'un jeune recrû forestier guyanais. Thèse de l'Université de Paris XI, $105 \mathrm{p}$

Lacoste JF, Alexandre DY (1989) Secondary forest dynamics and management following paper pulp cutting in French Guiana. Interciencia 14 (6), 323-328 
Lacoste JF, Alexandre DY (1990) Effet d'un dégagement précoce sur la valorisation syvicole du recrû après coupe papetière en Guyane française. Atelier MAB, Cayenne mars $1990,10 p$

Lemée A (1953) Flore de la Guyane française. Lechevalier, Paris, Vol 2, $398 \mathrm{p}$

Lescure JP (1986) La reconstitution du couvert végétal après agriculture sur brulis chez les Wayapi du haut Oyapock (Guyane française). Thèse Université Paris VI, $142 p$

Leslie AJ (1987) Aspects économiques de l'aménagement des forêts tropicales. Unasylva 155 (39), 46-58

Loureiro AA, Silva MF da (1968) Catalogo das madeiras da Amazonia. Sudam, Belem, $433 p$

Miquel S (1987) Morphologie fonctionnelle de plantules d'espèces forestières du Gabon. Bull Mus Natl Hist Nat, Sect B, Adansonia Bot Phytochim 9, 101-121

Oldeman RAA (1974) L'architecture de la forêt guyanaise. Mémoire Orstom, $n^{\circ} 73$, Paris, $204 p$

Pedroso LM, Pereira AP (1971) Informaçoes preliminares sobre a silvicultura de 38 especies florestais da estaçao experimental de Curua-Una. Sudam, Belem, $39 \mathrm{p}$

Pereira AP, Pedroso LM (1972) Experimentos de silvicultura tropical. Sudam, Belem, $82 \mathrm{p}$

Prévost MF (1981) Mise en évidence de graines d'espèces pionnières dans le sol de la forêt primaire en Guyane. Turrialba 31 (1), 121-127
Prévosi MF (1983) Les fruits et les graines des espèces végétales pionnières de Guyane française. Rev Écol Terre Vie 38 (2), 121-145

Rollet $B$ (1969) La régénération naturelle en forêt dense humide sempervirente de la plaine de la Guyane vénézuélienne. Bois For Trop 124, 19-38

Rollet B (1983) La régénération naturelle dans les trouées. Bois For Trop 201, 3-34

Roth I (1972) Estructura anatomica de la corte$z a$ de algunas species arboreas venezolanas de Celastraceae. Acta Bot Venez 7 (1, 2, 3, 4), $83-100$

Sabatier D (1983) Fructification et dissémination en forêt guyanaise : l'exemple de quelques espèces ligneuses. Thèse de $3^{e} \mathrm{cycle}$, ESTL, Montpellier, $338 p$

Schulz JP (1960) Ecological studies on rain forest in northern Surinam. Verh $K$ Ned Akad Wet Afd Natuurkd Tweede Reeks, $367 \mathrm{p}$

Sudam (1979) Pesquisas e informaçoes sobre espécies florestais da Amazonia. Departemento de recursos naturais, Centro de tecnologia Madeira, Belem, $111 \mathrm{p}$

Thiel J (1983) Fiche de reconnaissance du Goupi. Bois For Trop 201, 48-49

Vink AT (1977) Surinam timbers. Surtim, Paramaribo, $170 \mathrm{p}$

Willis JC (1973) A dictionary of flowering plants and ferns. Cambridge University Press, $8^{e}$ édition, $1245 p$ 